\title{
An Inertia Driven Micro-Actuator for Space Applications
}

\author{
MAKOTO MITA, ${ }^{1}$ MANABU ATAKA, ${ }^{1}$ HIROYUKI FUJITA, ${ }^{2}$ and HIROSHI TOSHIYOSHI ${ }^{3}$ \\ ${ }^{1}$ Inst. of Space, Astronautical Science, Japan Aerospace Exploration Agency, Japan \\ ${ }^{2}$ University of Tokyo, Tokyo, Japan \\ ${ }^{3}$ Research Center for Advanced Science, Technology, University of Tokyo, Tokyo, Japan
}

\begin{abstract}
SUMMARY
We have developed an inertia-driven micro actuator for the harsh environment of space. Different from the conventional PZT-driven or other actuators, the newly developed electrostatic mechanism is free from material property change due to temperature. A mass suspended in the actuation system is designed to collide with the internal wall to produce thrust by an impact-type inchworm mechanism. (c) 2014 Wiley Periodicals, Inc. Electron Comm Jpn, 97(3): 60-67, 2014; Published online in Wiley Online Library (wileyonlinelibrary.com). DOI 10.1002/ecj. 11502
\end{abstract}

Key words: inertia-driven actuator; space applications.

\section{Introduction}

Primarily motors and piezo elements have been used conventionally as drive devices for space probe mechanisms. However, planets located close to the Sun, such as Mercury and Venus, have high temperature environments of several hundred degrees Celsius, while on the other hand, cryogenic environments of tens to hundreds of degrees below $0{ }^{\circ} \mathrm{C}$ may exist in deep space or on the dark side of astronomical bodies located far from the sun. In such high temperature or cryogenic environments, motors and piezo elements show drastic degradations in performance, or even fail in the worst case. Electrostatically driven actuators are suitable for such extreme environments. Such actuators use air-gaps as dielectrics, and their operating characteristics theoretically do not depend on temperature. This means that drive systems with superior environmental tolerance can be implemented. However, the output of electrostatically driven actuators is low compared to that of magneticallydriven or piezoelectrically driven units. In order to re- solve this problem, we developed an inertia-driven actuator featuring a high output $[1,2]$. However, in the previous research the output was not sufficiently high to implement the actuator on a printed board rather than on a single chip. In this study we report improvements that result in a greater driving force.

\section{Driving Principle}

The working principle of the proposed device is illustrated in Fig. 1. Here the driving voltage is applied to the drive electrode and the stopper is set to the same potential as the mass (grounded) so as to avoid short circuiting.

(a) First, the driving voltage is applied between the movable mass and the stopper (actually, the drive electrode). The mass is accelerated toward the stopper by an electrostatic attractive force. (b) When the mass collides with the stopper, the whole chip moves due to the impact force. (c) When the voltage is removed, the mass returns to its original position due to the restoring force of the spring (suspension). The chip moves forward as operating sequence (a) to (c) is repeated. The travel distance due to one collision is small, but as the collisions are repeated, the chip can move over a long distance similarly to an inchworm.

\section{Operation Analysis and Simulation}

Aiming at operation analysis for the purpose of design, we determine the collision velocity of the movable mass, which has a strong effect on the driving force generated by impact. A simplified model of the device used in analysis is shown in Fig. 2. In this model, a collision phenomenon is represented by a stiff virtual spring applying pressure to a wall [3]. Here $k$ is the spring constant of the suspension supporting the mass, $k_{w}$ is the spring constant of the virtual spring, $m$ is the mass, $g$ is the initial gap, and $V_{d}$ is the driving voltage. 


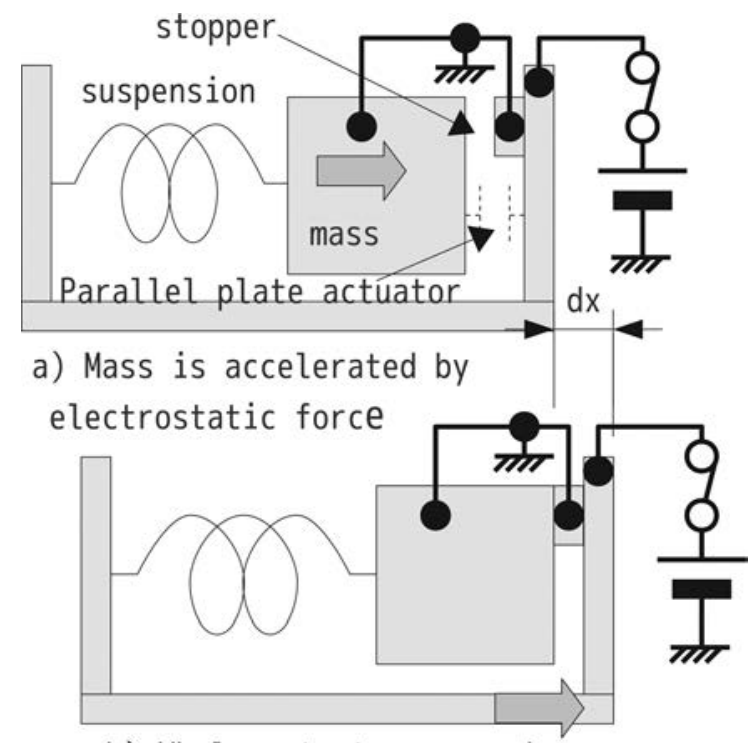

b) Whole actuator moves by impact force

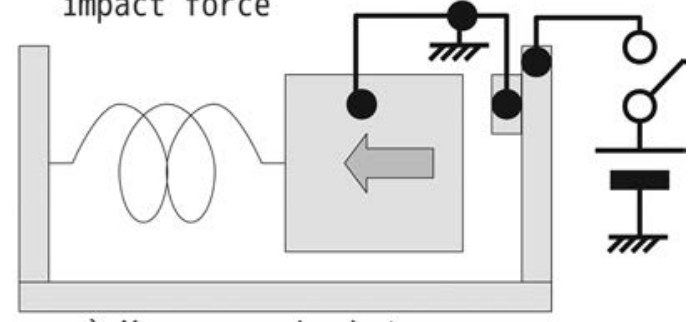

c) Mass goes back to initial position

Fig. 1. Principle of actuator.

In the initial state (time $t=0$, displacement $x=0$ ), the system energy $W_{0}$ includes only electrostatic energy, that is,

$$
W_{0}=\frac{1}{2} C_{0} V_{d}^{2} .
$$

Here $C_{0}$ is the electrostatic capacitance of the parallel plate actuator in the initial state. Immediately before collision, the system energy $W_{f}$ can be expressed as follows:

$$
W_{f}=\frac{1}{2} C_{f} V_{d}^{2}+\frac{1}{2} m v_{f}^{2}+\frac{1}{2} k x_{f}^{2},
$$



Fig. 2. Model of actuator. where $C_{f}$ is the electrostatic capacitance of the parallel plate actuator immediately before collision, $x_{f}$ is the displacement of the mass at collision, and $m$ is the mass. In addition, according to the law of conservation of energy, the work $W$ of the power source equals the difference between $W_{f}$ and $W_{0}$, and therefore,

$$
W=\Delta Q V_{d}=\Delta C V_{d}^{2}=\left(C_{f}-C_{0}\right) V_{d}^{2}=W_{f}-W_{0},
$$

where $\Delta C$ is the difference in electrostatic capacitance between the initial state and that immediately before collision. Now the following can be obtained by substituting Eqs. (1) and (2) into Eq. (3):

$$
\frac{1}{2} m v_{f}^{2}=\frac{1}{2}\left(C_{f}-C_{0}\right) V_{d}^{2}-\frac{1}{2} k x_{f}^{2} .
$$

Thus the velocity $v_{f}$ immediately before collision is derived as follows:

$$
v_{f}=\sqrt{\frac{1}{m}\left(C_{f}-C_{0}\right) V_{d}^{2}-k x_{f}^{2}} .
$$

In addition the electrostatic capacitance $C_{0}$ in the initial state and $C_{f}$ immediately before collision can be expressed as follows:

$$
\begin{aligned}
C_{0} & =\frac{\varepsilon_{0} S}{g} \\
C_{f} & =\frac{\varepsilon_{0} S}{g-x_{f}} .
\end{aligned}
$$

Here $\varepsilon_{0}$ is the permittivity of a vacuum and $S$ is the area of the parallel plates. Since $C_{f}$ represents the capacitance immediately before collision, deformation of the spring $k_{w}$ (Fig. 2) after collision is not considered. The following is obtained by substituting the above equations into Eq. (5):

$$
v_{f}=\sqrt{\frac{1}{m} \varepsilon_{0} S\left(\frac{1}{g-x_{f}}-\frac{1}{g}\right) V_{d}^{2}-k x_{f}^{2}} .
$$

In the real device, the driving voltage is $V_{d}=150 \mathrm{~V}$, the mass is $m=20 \mathrm{mg}$, the initial gap is $g=20 \mu \mathrm{m}$, the area of the parallel plate actuator is $S=2.4 \times 10^{-7} \mathrm{~m}^{2}$. and the gap immediately before collision is $\left(g-x_{f}\right)=2 \mu \mathrm{m}$. Thus the displacement $x_{f}$ immediately before collision is $18 \mu \mathrm{m}$. Therefore, the energy $k x_{f}^{2}$ accumulated in the spring is smaller than the first term by about three orders of magnitude and thus can be ignored. In addition $x_{f}$ is usually made as close to the gap $g$ as possible, so that $1 / g$ is negligibly small compared to $1 /\left(g-x_{f}\right)$. Thus Eq. (7) can be simplified as follows:

$$
v_{f}=\sqrt{\frac{1}{m} \frac{\varepsilon_{0} S V_{d}^{2}}{g-x_{f}}} .
$$

Substituting the real device parameters into the above equation, we obtain $v_{f}=0.035 \mathrm{~m} / \mathrm{s}$.

In order to test the operation of the device, we carried out simulations using an analysis of electrostatic actuators 


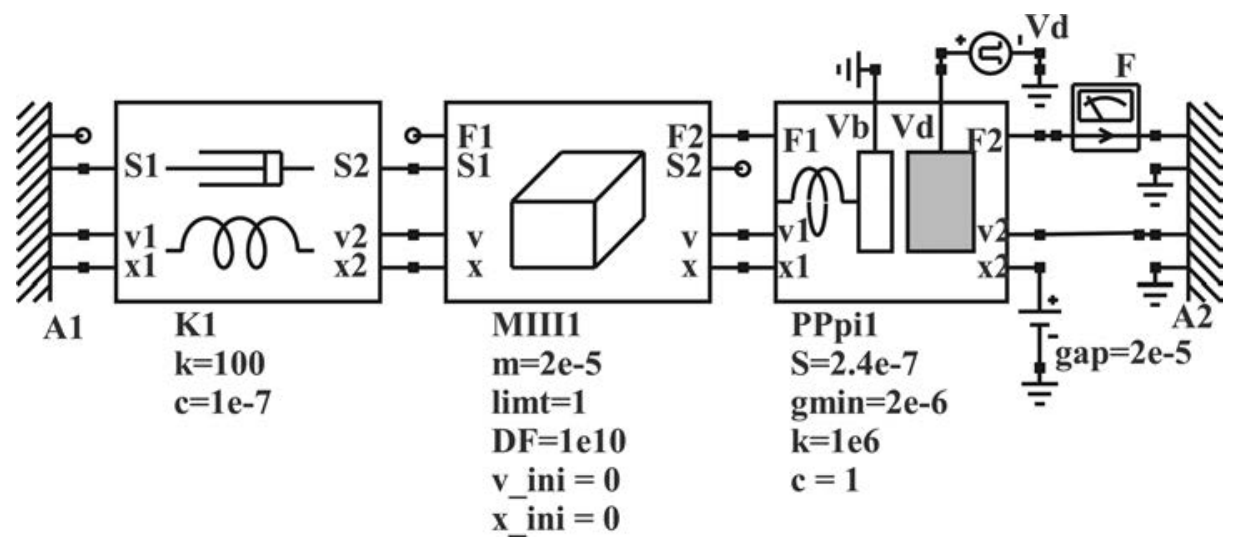

Fig. 3. Simulation model of actuator.

based on a circuit simulator that was proposed earlier by our group $[4,5]$. The device model in Fig. 2 was modified for the simulations as shown in Fig. 3. In the diagram, $\mathrm{K} 1$ is suspension module supporting the movable mass, MIII1 is the equation-of-motion solver, and PPpi1 is the parallel plate actuator module, including the virtual spring that simulates collision. As explained above, collision is modeled by application of pressure to a wall by a spring with an extremely large spring constant $\left(10^{6} \mathrm{~N} / \mathrm{m}\right.$ in the simulations). Using this model, we confirmed the conservation of momentum prior to the simulations. However, these simulations did not involve forward movement due to the impact force.

The simulation results are presented in Fig. 4. The diagrams represent (a) the mass displacement, (b) the velocity, and (c) the impact force. As can be seen from the

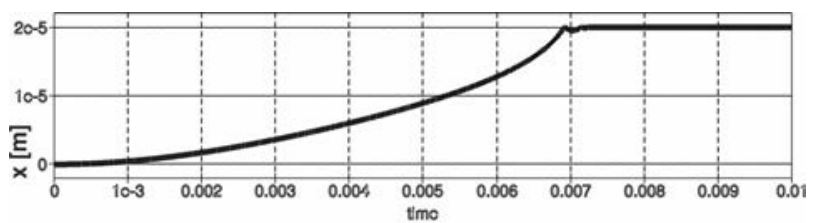

a) Displacement

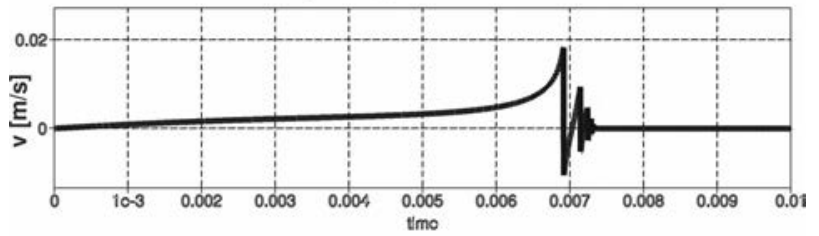

b) Velocity

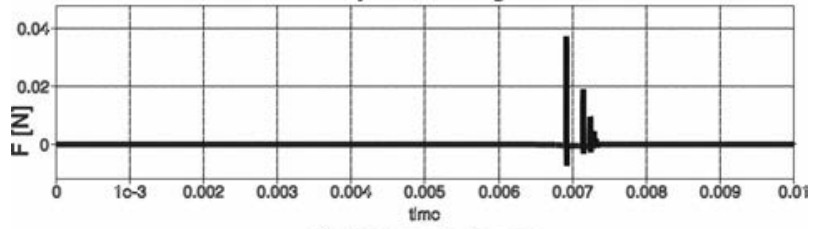

c) Impact force

Fig. 4. Simulation results. diagrams, the collision phenomenon is realistically analyzed. In addition the velocity at collision was 0.02 $\mathrm{m} / \mathrm{s}$, which is close to the value calculated by Eq. (8). The instantaneous impact force produced by collision was $15 \mathrm{mN}$ to $35 \mathrm{mN}$, which is about 1000 times the force of several $\mu \mathrm{N}$ or several tens of micro Newtons generated by conventional electrostatic microactuators. In these simulations, we investigated how the impact force and velocity were affected by various parameters, including the mass $m$, spring constant $k$, and initial gap $g$. We found that a greater mass $m$ contributes strongly to the impact force. As follows from Eq. (8), $v_{f}$ decreases with increasing mass; however, this decrease can be compensated by a greater driving force. That is, the driving voltage and the parallel plate area should be increased. These results agree with the above analysis of the velocity before collision.

Figure 5 shows the simulation results for operation at the resonant frequency obtained with the same model. The diagrams represent (a) the mass displacement, (b) the velocity, (c) the impact force, and (d) the displacement and velocity on an expanded time axis. In diagram (d), the dashed line represents the displacement, shown on the left axis, and the solid line represents the velocity, shown on the right axis. When the device is operated at the resonant frequency, the amplitude gradually increases and eventually the mass collides with the stopper. At collision, the waveforms are disturbed but essentially remain sinusoidal. The real device parameters were used in these simulations, and the obtained collision velocity $(0.03 \mathrm{~m} / \mathrm{s})$ was close to that in Fig. 4 $(0.02 \mathrm{~m} / \mathrm{s})$. When the spring constant is set higher, the vibration velocity increases and collision velocity likewise grows. Therefore, in the case of resonant driving, the vibration velocity can be greater than that obtained by Eq. (8) if the spring constant $k$ is sufficiently large, that is, if the resonant frequency is sufficiently high. In addition the collision rate per unit time grows, resulting in a higher travel speed. Thus, a greater driving force and a higher travel speed than in single collisions can be obtained by driving at the resonant frequency. 


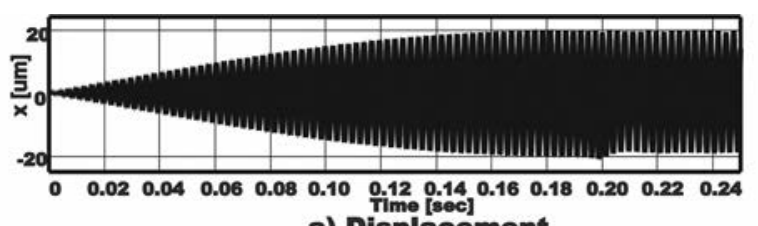

a) Displacement

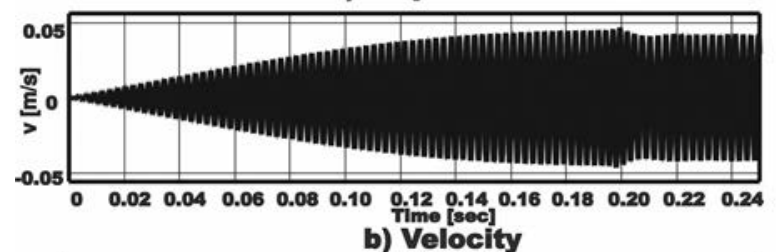

b) Velocity

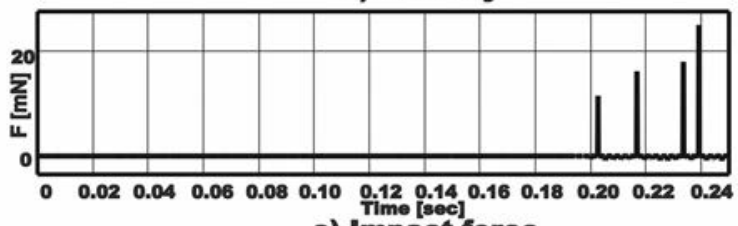

c) Impact force

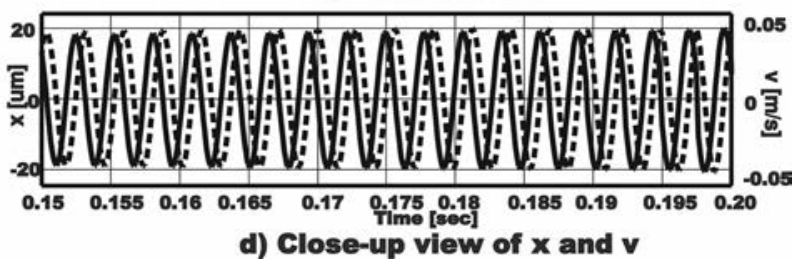

Fig. 5. Simulation results.

The simulation results showed that in case of resonant operation, a sinusoidal approximation is acceptable, and therefore we describe motion of the mass as follows:

$$
x(t)=A \sin (\omega t) .
$$

Here $x(t)$ is the mass displacement, $A$ is the amplitude, and $\omega$ is the angular frequency. The mass velocity $v(t)$ is obtained by time differentiation of $x(t)$ :

$$
v(t)=A \omega \cos (\omega t) .
$$

Because operation at the resonant frequency is assumed, the angular velocity $\omega$ is can be found from the spring constant $k$ and mass $m$ as follows:

$$
\omega=\sqrt{\frac{k}{m}} .
$$

The vibration is limited by the stopper, and hence $A \# \sim g$. Substituting this relation into Eq. (10), the maximum mass velocity $v_{\text {max }}$ at resonance is

$$
v_{\max }=g \sqrt{\frac{k}{m}} .
$$

When the driving voltage is sufficiently high, the collision velocity approaches $v_{\max }$; thus this value can be used in design.

From the above results it follows that the collision velocity can be increased by setting the initial gap, spring constant, and driving speed as large as possible. The mass velocity is greatest at a displacement $x=0$, and therefore the maximum collision velocity is not equal to $v_{\max }$ calculated above. However, an increase in $v_{\max }$ results in a higher collision velocity, and thus we set $v_{\max }$ high in design.

Now the impact force $F_{c}$ produced at collision can be expressed in terms of the collision time $\Delta t$ and the velocity change $\Delta V$ as follows:

$$
F_{c}=m \frac{\Delta V}{\Delta t} .
$$

That is, the impact force varies directly with the mass $m$. The driving voltage is limited by the dielectric strength of the device, which depends on the material and the structure. The initial gap and the drive electrode area are limited by the device dimensions. With these limitations in mind, we designed the device as explained below.

\section{Design and Structure}

The structure of the actuator is shown in Fig. 6. The driven mass is supported by an outer frame consisting of

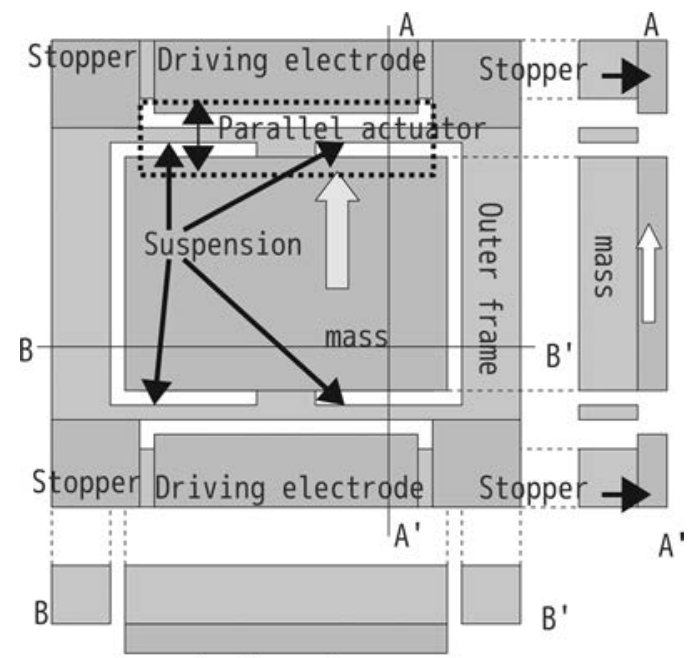

a) Top view

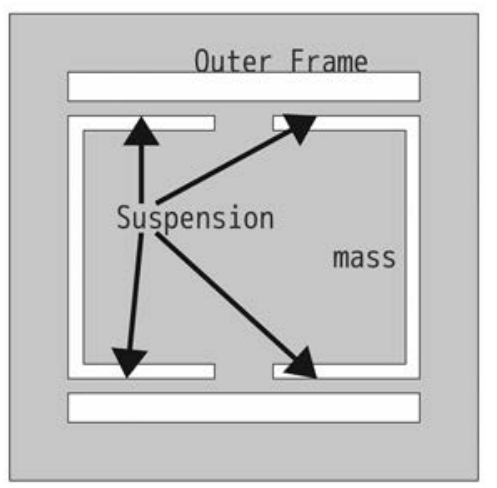

b) Bottom view

Fig. 6. Structure of device. 
four springs. This device is driven by a parallel plate electrostatic actuator. The bottom layer of the SOI substrate provides a mechanical structure including the suspension and mass, and the top layer acts as a parallel plate actuator. The electrodes placed on the mass are grounded via cantilevers to the bottom level [6].

As explained above, a large mass and a high collision velocity, that is, high $v_{\max }$, are required to obtain a strong impact force. For this purpose, the spring constant and gap should be set large, as suggested by Eq. (12). In addition, not only the impact force at collision but also the collision rate per unit time should be increased to obtain a higher travel speed of the whole device. In this study, a large portion of the chip area is allocated to the mass in order to implement a large mass. In addition there is a limit to the dielectric strength of the device, and therefore, after determination of the driving voltage, the drive electrode area is set as large as possible so as to obtain a stronger electrostatic attractive force at the given voltage. The spring constant too should be set as high as possible in order to increase the collision velocity and the collision rate per unit time; on the other hand, operation must be restricted to the abovementioned range of the driving force. In the case of driving at the resonant frequency, the amplitude grows by a factor equal to the $Q$-factor compared to DC driving, and

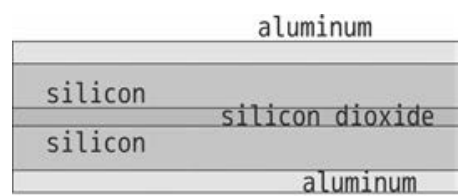

a) Aluminum deposition

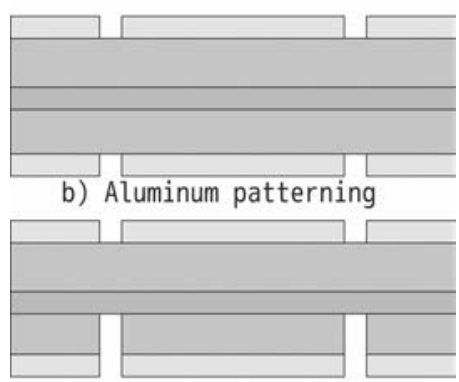

c) Deep RIE from backside

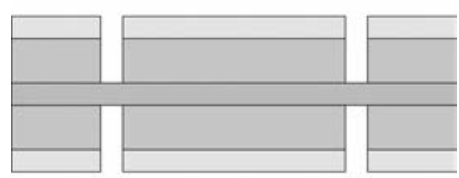

d) Deep RIE from front side

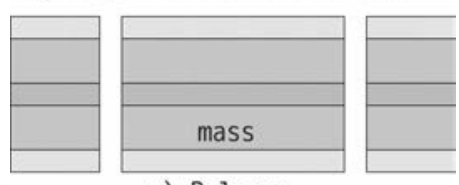

e) Release

Fig. 7. Fabrication process. therefore the gap $g$ is set within $Q$ times the displacement determined by the spring constant and the driving force. From the above considerations, we determined the mass, driving voltage, and spring constant and fabricated a prototype device.

\section{Fabrication Process}

The device was fabricated on an Silicon On Insulator (SOI) substrate. The SOI substrate had an active layer $80 \mu \mathrm{m}$ thick, a silicon oxide film layer (BOX layer) $2 \mu \mathrm{m}$ thick, and a handle layer $300 \mu \mathrm{m}$ thick. Stable results were assured by using a simple fabrication process, as shown in Fig. 7. First, (a) aluminum was vacuum-deposited on both

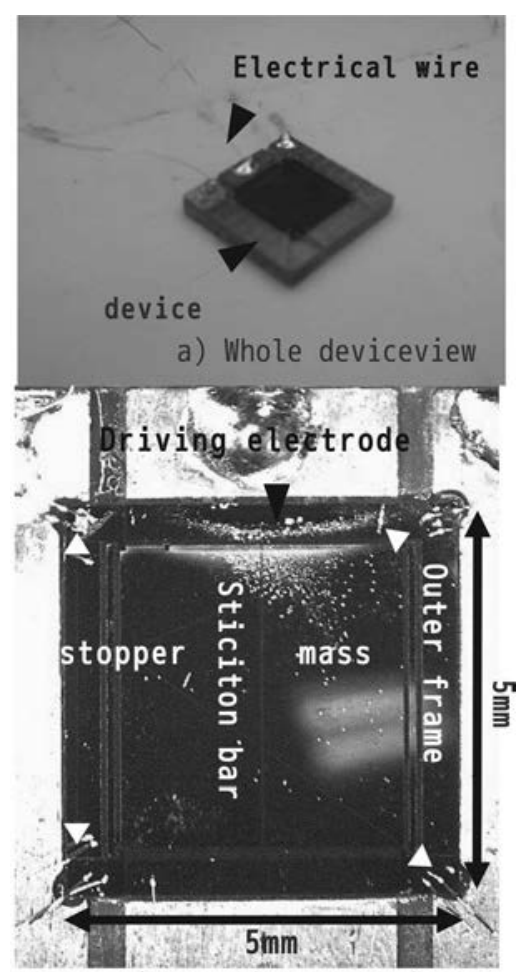

b) Top view of device

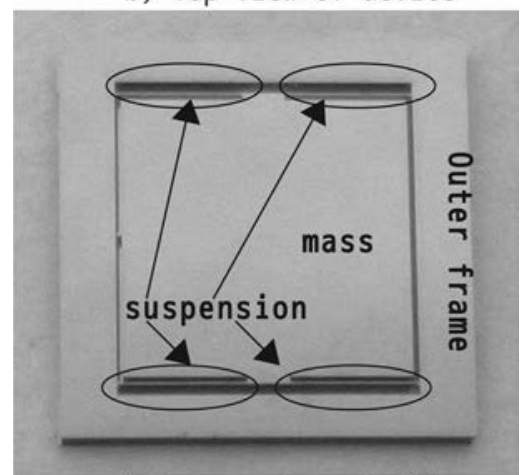

c) Bottom view of chip

Fig. 8. Photograph of device. 
sides of the substrate to form the etching mask. Then, (b) the pattern was transferred to the resist using the photomask, the deposited aluminum was etched, and a mask pattern for silicon etching was created. After that, (c) silicon was deepetched from the backside using aluminum as the etching mask, thus forming the outer frame, mass, and suspension on the bottom layer (handle layer). Then, (d) deep etching with the aluminum mask was performed in the same way from the front side to form the electrostatic actuator on the top layer (active layer). Finally, (e) the silicon oxide film was removed from between the silicon layers.

\section{Fabrication Results}

The fabricated device is shown in Fig. 8. The device size was $5 \mathrm{~mm} \times 5 \mathrm{~mm}$ and the thickness was about $0.4 \mathrm{~mm}$. The suspension spring was $15 \mu \mathrm{m}$ wide, $1.5 \mathrm{~mm}$ long, and $300 \mu \mathrm{m}$ high. The size of the mass was $3 \mathrm{~mm} \times 3 \mathrm{~mm}$ and the width of the outer frame was $0.5 \mathrm{~mm}$. The mass and the outer frame had the same thickness, and therefore the mass ratio was equal to the area ratio. The mass constituted $50 \%$ of the entire device. The size of the PC board was $8 \mathrm{~mm} \times$ $8 \mathrm{~mm}$; copper wires $0.05 \mathrm{~mm}$ in diameter were soldered to the board in order to supply the driving voltage.

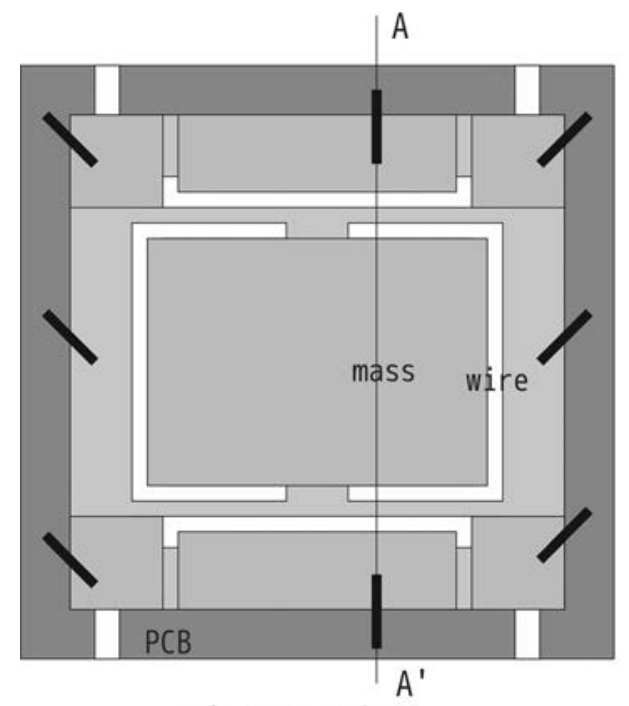

a) Top view

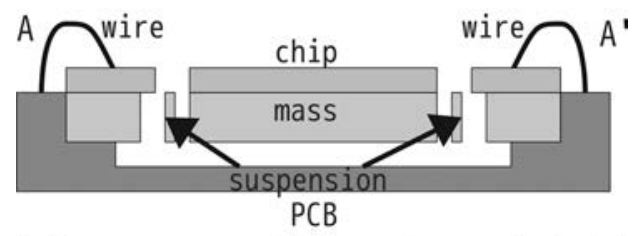

b) cross-section view at $A-A^{\prime}$

Fig. 9. Cross-section view of device.
The layout of the printed board is shown in Fig. 9. In order to connect the device to the power supply, the fabricated chip was mounted on a special printed circuit board, and power supply from the board was implemented by wire bonding. (In Fig. 9, the mass module does not contact the printed circuit board.)

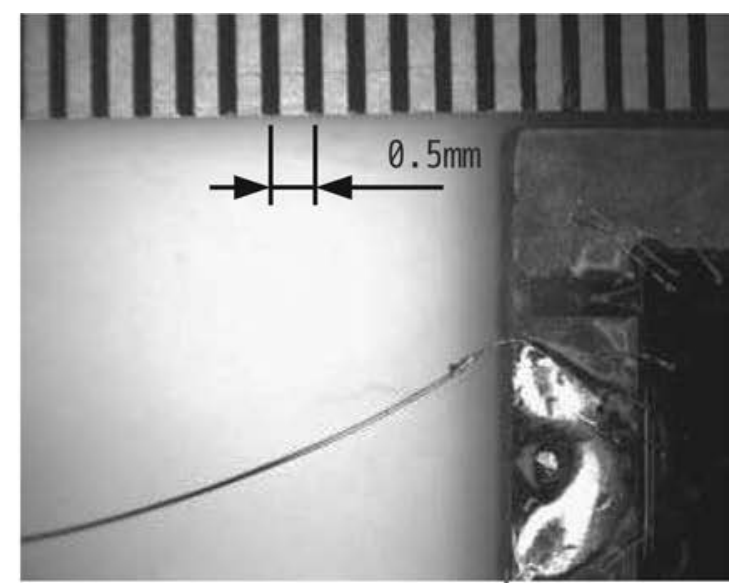

a) Initial position

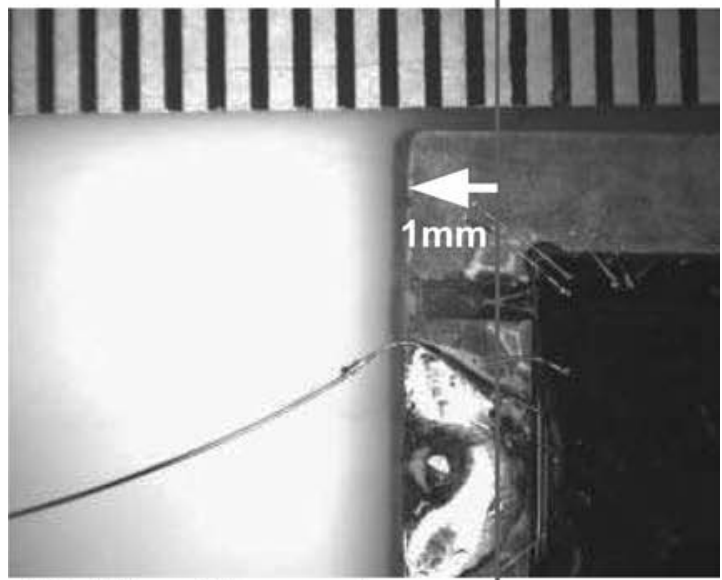

b) After 10 seconds |IIIIIIIIIIIIIII

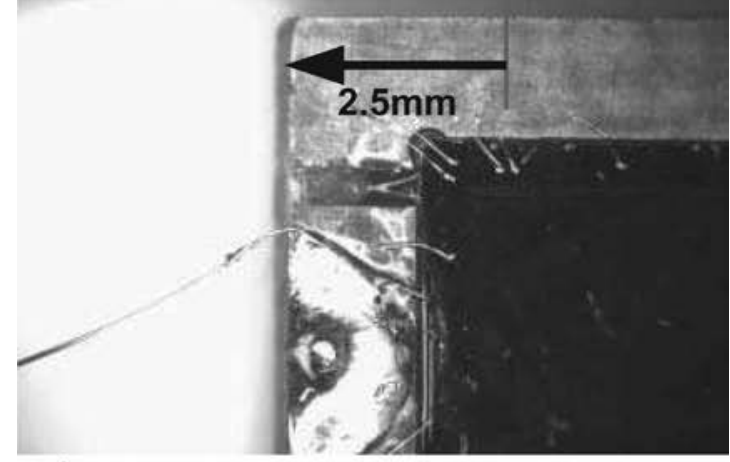

c) After 20 seconds

Fig. 10. Operation photographs. 


\section{Driving Results}

In operation tests with a sinusoidal driving voltage, the resonant frequency of the device was $311 \mathrm{~Hz}$. The driving results of the whole chip are illustrated in Fig. 10. The top, middle, and bottom photographs were taken, respectively, before actuation began, $10 \mathrm{~s}$ after actuation began, and $20 \mathrm{~s}$ after actuation began. Using thin copper wires, a sinusoidal voltage with an amplitude of $150 \mathrm{~V}$, a bias of $75 \mathrm{~V}$, and a frequency of $311 \mathrm{~Hz}$ was applied to the device mounted on the printed board. We found that the whole printed board including the chip traveled a distance of about $1 \mathrm{~mm}$ in $10 \mathrm{~s}$ while resisting frictional and restoring forces. Thus, the device traveled about $100 \mu \mathrm{m}$ per second; considering the driving frequency of $311 \mathrm{~Hz}$, the average travel distance per collision can be estimated as about $320 \mathrm{~nm}$.

The total weight including the printed board was about $0.2 \mathrm{~g}$, and the weight of the chip was about $20 \mathrm{mg}$. Thus the device is capable of driving about 10 times its own weight. Inclining the stand on which the device was placed, we found that the device started off at an angle of about $22^{\circ}$. Thus, the coefficient of static friction between the device and the stand can be estimated as about 0.37 . Considering the frictional force calculated with this coefficient as well as the restoring force of the copper wire, the driving force was of $\mathrm{mN}$ order. This is an order of magnitude smaller than the simulation value; however, we can expect to achieve more adequate simulations by making more accurate measurement of the collision time $\Delta t$ and the actual collision velocity.

\section{Conclusions}

We considered an inertia-driven micro-actuator for space applications. In particular, we succeeded in impact driving of an entire chip including the printed circuit board, which was difficult with the previous MEMS actuator that produced driving force of about $10 \mu \mathrm{N}$. In the future, we plan to continue development aiming at a further increase in the driving force. In addition, we are planning to carry out operation tests in high-temperature and cryogenic environments in order to improve the for space applications.

\section{REFERENCES}

1. Mita M, Arai M, Tensaka S, Kobayashi D, Fujita H. A micromachined impact microactuator driven by electrostatic force. J Microelectromech Syst 2003;12(1):3741.

2. Mita M, Takahashi K, Ataka M, Fujita H, Toshiyoshi H. Highly mobile 2D micro impact actuator for space applications. The 14th Int Conf on Solid-State Sensors, Actuators and Microsystems (Transducers '07), Lyon, France; (2EC13.P) 2007.

3. Faik S, Witteman H. Modeling of impact dynamics: A literature survey. 2000 International ADAMS User Conference; 2000.

4. Mita M, Toshiyoshi $\mathrm{H}$. An equivalent-circuit model for MEMS electrostatic actuator using open-source software Qucs. IEICE Electr Express 2009;6(5):256-263.

5. Mita M, Maruyama S, Yi Y, Takahashi K, Fujita H, Toshiyoshi H. Multi-Physics analysis for micro electromechanical systems based on electrical circuit simulator. IEEJ Trans Electr Electrc Eng 2011;6(2):180-189.

6. Takahashi K, Mita M, Fujita $\mathrm{H}$, Toshiyoshi $\mathrm{H}$. Switched-layer design of SOI bulk micromachined XYZ stage using stiction bar for interlayer electrical connection. IEEE J Microelectromech Syst 2009;18(4):818-827. 